\title{
Voluntary physical activity and leucine correct impairments in muscle protein synthesis in partially pancreatectomised rats
}

\author{
A. S. Serino • O. A. Adegoke $\cdot$ S. Zargar \\ C. S. Gordon • A. A. Szigiato • T. J. Hawke • \\ M. C. Riddell
}

Received: 5 May 2011 / Accepted: 2 August 2011 /Published online: 10 September 2011

(C) Springer-Verlag 2011

\begin{abstract}
Aims/hypothesis Poorly controlled type 1 diabetes mellitus can cause reduced skeletal muscle mass and weakness during adolescence, which may affect long-term management of the disease. The aim of this study was to determine whether regular voluntary physical activity and leucine feeding restore rates of protein synthesis and deficits in skeletal muscle mass in a young, hypoinsulinaemic/hyperglycaemic rat model of diabetes.

Methods Four-week-old male Sprague-Dawley rats were partially pancreatectomised (Px) to induce hypoinsulinaemia/hyperglycaemia and housed with/without access to running wheels for 3 weeks ( $n=12-14 /$ group). Sham surgery rats (shams) served as sedentary controls $(n=18)$. Protein synthesis and markers of protein anabolism were assessed in the fasted state and following leucine gavage.
\end{abstract}

O. A. Adegoke and M. C. Riddell share senior authorship on this manuscript.

A. S. Serino · O. A. Adegoke · S. Zargar · C. S. Gordon •

A. A. Szigiato $\cdot$ M. C. Riddell

School of Kinesiology and Health Science, York University,

4700 Keele Street,

Toronto, ON, Canada

A. S. Serino $\cdot$ O. A. Adegoke $\cdot$ S. Zargar $\cdot$ C. S. Gordon $\cdot$

A. A. Szigiato $\cdot$ T. J. Hawke $\cdot$ M. C. Riddell $(\bowtie)$

Muscle Health Research Centre, York University,

4700 Keele Street,

Toronto, ON M3J 1P3, Canada

e-mail: mriddell@yorku.ca

T. J. Hawke

Department of Pathology and Molecular Medicine,

McMaster University,

Hamilton, ON, Canada
Fibre type and cross-sectional areas of the gastrocnemius muscle were measured using a metachromatic ATPase stain. Results Compared with sedentary behaviour, regular activity lowered fasting glycaemia and reduced fed hyperglycaemia in Px rats. Active-Px rats, which ran $2.2 \pm 0.71 \mathrm{~km} / \mathrm{night}$, displayed greater muscle mass and fibre areas similar to shams, while sedentary-Px rats displayed a $20-30 \%$ loss in muscle fibre areas. Muscle protein synthesis (basal and in response to leucine gavage) was impaired in sedentary-Px (by $\sim 65 \%$ ), but not in active-Px rats, when compared with shams. Following leucine gavage, the phosphorylation status of eIF4E binding protein 1 (4E-BP1) and ribosomal S6 kinase 1 (S6K1), markers of mammalian target of rapamycin complex 1 (mTORC1) signalling, increased in shams (by two- and ninefold, respectively) and in active-Px (1.5- and fourfold, respectively) rats, but not in sedentary-Px rats.

Conclusion/interpretation Moderate physical activity in young Px rats normalises impairments in skeletal muscle growth and protein synthesis. These findings illustrate the critical compensatory role that modest physical activity and targeted nutrition can have on skeletal muscle growth during periods of hypoinsulinaemia in adolescent diabetes.

Keywords Adolescence - Exercise · Growth · Leucine · Muscle $\cdot$ Pancreatectomy $\cdot$ Physical activity $\cdot$ Protein synthesis $\cdot$ Rodents $\cdot$ Running wheel $\cdot$ Type 1 diabetes

$\begin{array}{ll}\text { Abbreviations } \\ \text { 4E-BP1 } & \text { 4E binding protein 1 } \\ \text { eIF } & \text { Eukaryotic initiation factor } \\ \text { mTORC1 } & \text { Mammalian target of rapamycin complex 1 } \\ \text { Px } & \text { Pancreatectomised } \\ \text { S6K1 } & \text { Ribosomal S6 kinase 1 } \\ \text { Akt } & \text { Serine/threonine protein kinase Akt }\end{array}$

Abbreviations

eIF Eukaryotic initiation factor

mTORC1 Mammalian target of rapamycin complex 1

Px Pancreatectomised

Akt Serine/threonine protein kinase Akt 


\section{Introduction}

Type 1 diabetes mellitus is associated with impairments in protein metabolism that can result in muscle wasting, impaired muscular development and a lifetime of reduced physical and functional capacities [1-3]. Altered protein metabolism in diabetes may be particularly evident in the paediatric population where optimal insulin therapy and glycaemic control are difficult to achieve [4, 5]. As such, the tissues of adolescent patients (i.e. cardiac and skeletal muscle, nerve, kidneys and blood vessels) are routinely exposed to protracted periods of hypoinsulinaemia/hyperglycaemia during critical periods of growth and development, which may impact the health and well-being of that child, either immediately or later in the disease progression [6]. However, little is known of how poor metabolic control affects skeletal muscle growth and development. Although the Diabetes Control and Complications Trial demonstrated that body mass was considerably lower in conventionally treated youth when compared with intensively treated youth, no body composition analysis was performed [4]. Compared with non-diabetic youth, adolescents with diabetes may be shorter, weaker and have a lower exercise capacity, particularly if they are under poor glycaemic control [3]. Insulin provision does not increase rates of whole body protein synthesis but rather lowers rates of protein degradation $[1,7]$. While these studies illustrate the effects of diabetes and insulin treatment on whole body protein metabolism in childhood, it is unclear if skeletal muscle protein metabolism is differentially affected by this disease.

Animal models of diabetes, although not a complete replication of the disease pathophysiology, may be a useful way for determining the effects of hypoinsulinaemia/hyperglycaemia on muscle growth and function. The partially pancreatectomised (Px) rat is one model of choice, as it mimics hypoinsulinaemia/hyperglycaemia, with some basal insulin production, which is similar to a child with diabetes at the time of diabetes diagnosis or a child who is receiving inadequate insulin administration. Poor glycaemic control in young rodent models of diabetes is associated with reduced muscle growth, function and repair, at least in sedentary cage conditions [3, 810]. Impairments in growth occur particularly in the type II/ glycolytic fibres, in part because of decreased rates of muscle protein synthesis [11-13] and probably through a reduced capacity of mammalian target of rapamycin complex 1 (mTORC1) signalling in response to protein intake [9]. Interestingly, insulin therapy in young streptozotocin-treated rat models of diabetes does not normalise muscle mass and muscle fibre areas, at least in the short term, perhaps because the diabetogenic agent itself arrests growth [14].

Interventions that increase muscle protein synthesis, such as resistance exercise, have been proposed as a means to help protect skeletal muscle in situations of acute or chronic muscle wasting [15]. Thus, the impairments in muscle growth and relative muscle wasting in poorly controlled diabetic rodent models may be diminished with increased physical activity. However, severely diabetic Px rat models do not display increased rates of muscle protein synthesis after acute resistance exercise [16, 17], perhaps because a permissive amount of insulin may need to be present [18]. In contrast, it may be that regular aerobic type exercise can increase muscle protein synthesis via insulin/ IGF-1 independent mechanisms [19]. Whether regular physical activity normalises muscle growth and protein metabolism in younger diabetic rat models is unknown.

The specific aims of this study were to determine whether voluntary daily physical activity: (1) increases muscle protein synthesis and muscle growth in young Px rats; and (2) alters the $\mathrm{mTORC} 1$ signalling response of skeletal muscle to acute leucine feeding.

\section{Methods}

Ethics statement

All experiments were approved by the York University Animal Care Committee in accordance with the Canadian Council for Animal Care guidelines.

\section{Animal characteristics}

Thirty-four young ( age 4 weeks, 45-55 g) male SpragueDawley rats were purchased from Charles River Laboratories (Montreal, QC, Canada). All animals had a 7 day habituation period to a $12 \mathrm{~h}$ light-dark cycle (lights on at 08:00 hours and lights off at 20:00 hours) in a temperature $\left(22-23^{\circ} \mathrm{C}\right)$ and humidity $(50-60 \%)$ controlled room. The animals (body mass 100-120 g) were then randomly assigned to one of three groups: sham operated (shams, $n=18$ ); sedentary pancreatectomised (sedentary-Px, $n=14$ ); and physically active pancreatectomised (active-Px, $n=12$ ).

\section{Pancreatectomy surgery}

Details of the partial Px surgery are described elsewhere [9]. Briefly, on the seventh day following their arrival (day 0), the animals underwent surgery to either remove $90 \%$ of their pancreas (sedentary-Px and active-Px groups) or to have the sham procedure, as previously described [9].

\section{Design}

The sham and sedentary-Px rats were housed two animals per cage and remained in traditional rodent cages for the 
entire duration of the study. On day 7 post surgery, activePx rats were separated and individually given $24 \mathrm{~h}$ access to a standard running wheel cage (Harvard Apparatus). Blood glucose levels were measured (09:00 hours) via tail nick three times a week to follow glycaemic levels. Body mass was also measured three times per week. Wheel revolutions were read daily and multiplied by the wheel circumference to obtain daily running distances. On day 26 post surgery, fed plasma samples were collected in heparinised tubes via tail nick at 08:00 hours for a basal corticosterone measurement. To confirm hypoinsulinaemia in the fed state in active and sedentary-Px rats relative to shams, and to confirm that regular activity did not change insulin secretory capacity, another group of identically treated rats underwent blood collection via saphenous vein bleed in a fed ( 9:00 hours) and fasted ( 20:00 hours) state on days 18 and 22 , respectively ( $n=5-7 /$ group). All animals were given standard rodent chow (Lab Diet 5012) and water ad libitum. On day 27, the animals were deprived of food for $\sim 18 \mathrm{~h}$. Half of the animals in each group (6-9 per group) were randomly selected and administered with a leucine solution by oral gavage, while the others received a water gavage (i.e. nutrient placebo group). Active-Px rats were pair-matched to account for differences in running distances. For the leucine gavage, standardised volumes of a leucine solution $(24 \mathrm{ml} / \mathrm{kg}$ of L-leucine solution in sterile water) were administered. This quantity of leucine $(0.48 \mathrm{~g} / \mathrm{kg})$ was calculated to be approximately $40 \%$ of what is consumed in a $24 \mathrm{~h}$ period by the shams [20]. For the water gavage, animals were administered an equivalent volume of sterile water to control for the stress response that is produced by gavage administration [21].

To measure muscle protein synthesis, we used the modified flooding dose technique [22], as described previously [9, 23]. For this, $20 \mathrm{~min}$ after the oral gavage, rats were restrained in a cloth blanket and given a flooding dose of radiolabelled phenylalanine $(1 \mathrm{ml} / 100 \mathrm{~g}$ body weight of $150 \mathrm{mmol} / \mathrm{l}$ $\mathrm{L}-\left[2,3,4,5,6-{ }^{3} \mathrm{H}\right]$ phenylalanine containing $1.85 \mathrm{MBq} / \mathrm{ml}$ ) intraperitoneally. After $10 \mathrm{~min}$, the animals were killed by decapitation. Blood was collected and gastrocnemius muscles were quickly removed, snap-frozen in liquid nitrogen, and stored at $-80^{\circ} \mathrm{C}$.

\section{Sample analyses}

Muscle protein synthesis For determination of protein synthesis, samples were analysed as described previously $[9,22,23]$. Fractional rates of protein synthesis $\left(k_{\mathrm{s}}\right)$ were determined using the formula $k_{\mathrm{S}}=\left(S_{\mathrm{B}} \times 100\right) /\left(S_{\mathrm{A}} \times t \times 0.9\right)$, where $t$ is the time interval between the time of injection and the freezing of the sample in liquid nitrogen expressed in days, $S_{\mathrm{B}}$ is the specific activity of phenylalanine in the protein bound amino acid pool and $S_{\mathrm{A}}$ is the specific activity of phenylalanine in the intracellular free amino acid pool. The factor of 0.9 was used to correct for the fact that the tracer was delivered by intraperitoneal injection rather than intravenously [24].

Akt and markers of mammalian target of rapamycin (mTORC1) signalling Phosphorylated and total eukaryotic initiation factor (eIF) 4E binding protein 1 (4E-BP1), ribosomal protein S6 kinase 1 (S6K1) and serine/threonine protein kinase Akt (Akt) protein levels in gastrocnemius samples were measured as previously described [9].

Plasma corticosterone and insulin determinations Plasma corticosterone levels were analysed from blood collected in the basal state (resting, fed) (09:00 hours) using a radioimmunoassay kit (\#07120102; MP Biomedicals, Solon, OH, USA). Plasma insulin levels were measured on day 18 in a fed state ( 09:00 hours), after a $10 \mathrm{~h}$ fast ( 20:00 hours) and after an overnight fast just before gavage on day 22 ( 09:00 hours) using a commercially available enzyme-linked immunosorbent assay kit (Ultra Sensitive Rat Insulin ELISA kit \#90060; Crystal Chem, Downers Grove, IL, USA). This kit reports a low range sensitivity, based on $5 \mu$ samples, between 17 and $1,102 \mathrm{pmol} / \mathrm{l}$, with an ultra-high sensitivity of $5 \mathrm{pmol} / 1$ if $100 \mu \mathrm{l}$ of sample is used. Based on pilot work using sham and Px rats (after the overnight fast), it was determined that a $10 \mu \mathrm{l}$ sample fell above the lower limit of detection $(17 \mathrm{pmol} / \mathrm{l})$, thus $10 \mu \mathrm{l}$ of sample was assayed and corrected for volume loaded. Fed insulin levels were diluted in half with sample diluent, as per the kit's instructions, so that they also fitted below the upper limit of detection. The insulin response to oral gavage was expressed as the change between pre and post gavage, as previously carried out by Crozier et al [20].

\section{Histochemistry}

To identify skeletal muscle fibre type, a metachromatic myosin ATPase stain was performed on $10 \mu \mathrm{m}$ thick cross sections of muscle using a modified Ogilvie and Feeback protocol [25], as previously described [9]. Average fibre area was quantified based on the average area of $>20$ fibres for each fibre type per image, with the experimenter being blinded to the group [8]. Quantification analysis was performed with Adobe Photoshop CS version 8.0 and reported in $\mu \mathrm{m}^{2}$. All images were acquired with a Nikon Eclipse 90i microscope and Q-Imaging MicroPublisher 3.3 RTV camera with Q-Capture software.

\section{Data analyses}

Carestream Molecular Imaging Software (Carestream Health, Rochester, NY, USA) was used to quantify signals 
for phosphorylated and total S6K1, Akt and 4E-BP1. Statistical analyses were completed using Statistica 7.0 statistical software (StatSoft, Tulsa, OK, USA), with $P<0.05$ as the criterion for statistical significance. All data are expressed as mean \pm SEM. Two-way factorial ANOVAs, followed by a Fisher post hoc test, were used for the comparisons of blood glucose, body mass, insulin, Akt, 4E-BP1 and S6K1 levels. A one-way ANOVA was used to compare fasted blood glucose, running distances, corticosterone levels, muscle mass, fibre area and protein synthesis. Again, a Fisher post hoc test was performed where appropriate.

\section{Results}

Blood glucose and body weight measurements

Seven days post surgery, both sedentary-Px and active-Px animals had elevated fed blood glucose levels compared with shams ( $p<0.05$; Fig. 1a). However, active-Px rats had lower fed blood glucose levels than sedentary-Px rats on days 10 , 14,17 and 21 (all $p<0.05$ ). Fasted blood glucose concentrations, measured only on day 28 of the experimental period, were elevated in sedentary-Px but not in active-Px rats compared with shams (both $p<0.05$; Fig. 1b). SedentaryPx and active-Px animals weighed less than shams starting on day 14 and continued to do so for the duration of the study ( $p<0.05$; Fig. 1c). No significant differences in body mass were observed at any time in the study between the sedentary-Px and active-Px groups. Running distances averaged 2,198 $\pm 712 \mathrm{~m}$ per night in the active-Px group during the 3 week experimental period (Fig. 2).

\section{Corticosterone and insulin levels}

At week 3 of treatment, both sedentary-Px and active-Px animals had elevated basal (fed) corticosterone levels when compared with the shams ( $p<0.05$; Fig. 3a). Although activePx rats had $\sim 30 \%$ higher corticosterone levels than sedentary-Px rats, this difference was not statistically significant $(p=0.13)$. As expected, fasted insulin concentrations were comparable across groups, while fed values were elevated only in the shams (Fig. 3b). Prior to gavage administration, overnight fasted insulin levels were also not significantly different among the three groups $(41 \pm 9 ; 19 \pm 3$; $36 \pm 7 \mathrm{pmol} / 1$ in the shams, sedentary-Px and active-Px rats, respectively, $p>0.05$ ). In response to water and leucine gavage, insulin levels increased in shams (both, $p<0.05$ ); however, no significant difference in response occurred between water and leucine gavage (Fig. 3c). In the Px animals (both active and sedentary), neither water nor leucine caused an insulin response (Fig. 3c).
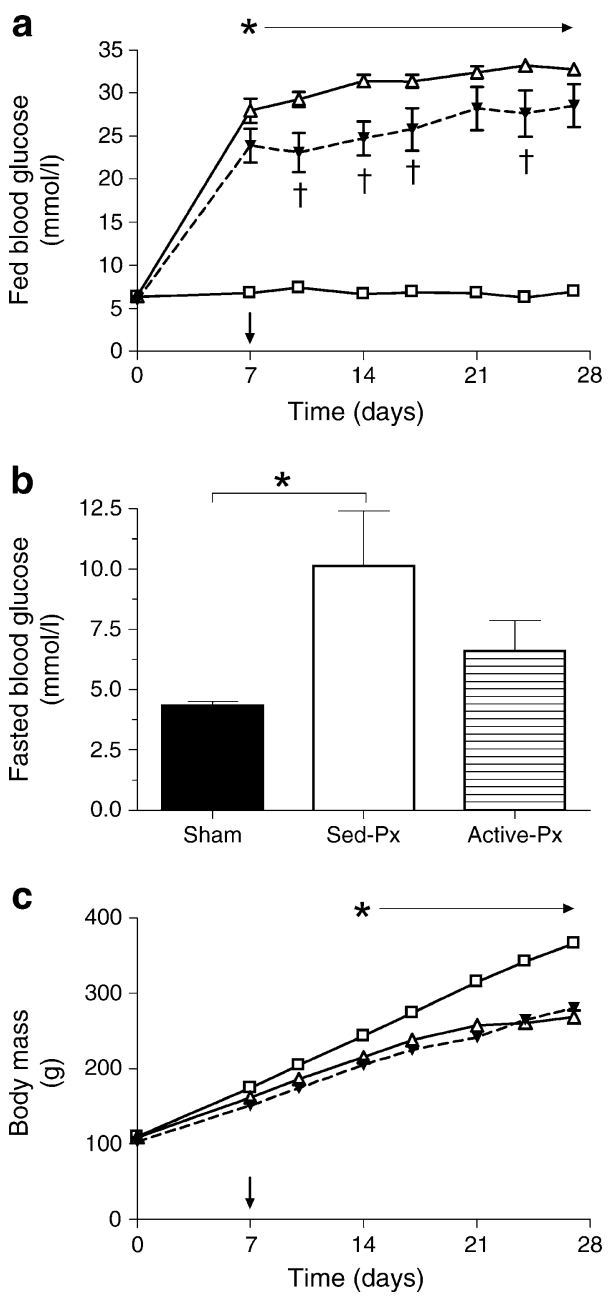

Fig. 1 Fed and fasted blood glucose levels and body masses of sham and Px rats. a Fed blood glucose concentrations were measured intermittently via tail nick in sham (white squares), sedentary-Px (white triangles) and active-Px (black triangles) groups, $n=9-12$ / group. b Fasted blood glucose concentration, as measured following an $18 \mathrm{~h}$ fast, $n=9-12$ /group.c Bi-weekly body mass in sham (white squares), sedentary-Px (white triangles) and active-Px (black triangles) rats, $n=9-12 /$ group. The arrow indicates the time of introduction of the running wheel for the active-Px group. ${ }^{*} p<0.05$ for difference between sedentary-Px and active-Px groups compared with shams. ${ }^{\dagger} p<0.05$ for active-Px vs sedentary-Px groups. Values are mean \pm SEM. Sed, sedentary

Skeletal muscle mass and fibre areas

Both the sedentary-Px and active-Px animals had smaller muscle mass than shams $(p<0.05)$, although active-Px animals had greater muscle mass than sedentary-Px animals $(p<0.05$; Fig. 4a). The same trend was observed when muscle mass was normalised for body weight ( $p<0.05$; Fig. 4b), indicating that some recovery of muscle growth took place in response to regular physical activity.

Histochemical analysis (Fig. 4c,d) revealed that the sedentary-Px group had reduced fibre cross-sectional areas in all fibre types compared with shams, with type IIb/IId 


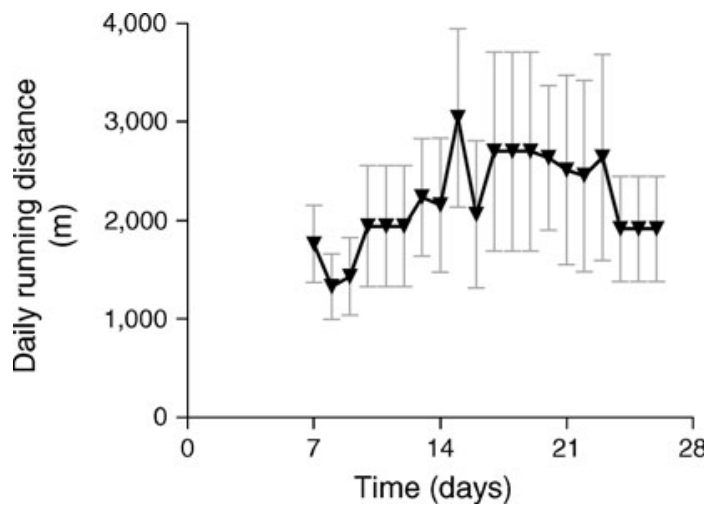

Fig. 2 Daily running distances in the active-Px rats. Values are mean \pm SEM

fibres being the most affected (group by fibre type interaction, $p<0.05$ ). Active-Px rats had similar fibre areas to shams in all fibre types.

Muscle protein synthesis

Sham animals had significantly higher muscle fractional rates of protein synthesis in the basal state (i.e. water gavage) when compared with sedentary-Px $(p<0.05)$ but not active-Px rats (Fig. 5). Comparisons between diabetic groups (active vs sedentary) revealed that exercise was effective in significantly increasing basal muscle protein synthesis $(p<0.05)$.

Compared with water, leucine gavage significantly increased muscle fractional rates of protein synthesis in shams $(p<0.05)$ and tended to increase protein synthesis in active-Px rats $(P=0.06)$. Leucine gavage had no significant effect on fractional rates of protein synthesis in the sedentary-Px animals. When comparing only animals that received a leucine gavage, the sham and the active-Px groups had significantly higher rates of muscle protein synthesis when compared with the sedentary-Px group $(p<0.05)$. Notably, fractional rates of protein synthesis in response to leucine were similar between shams and active-Px groups. There was no significant relationship between the running distance in the evening before gavage and the fractional rate of protein synthesis in the active-Px group $\left(r^{2}=0.16, p=0.44\right)$.

\section{Akt and markers of mTORC1 signalling}

Levels of Akt phosphorylation were measured at serine 473 and threonine 308 (Fig. 6a and b, respectively). No significant differences were observed between any of the groups, regardless of gavage treatment, in either of these markers of Akt activation. There was a tendency for leucine gavage to increase Akt phosphorylation at serine 473 in shams, but this increase did not achieve statistical signifi- cance $(p=0.26)$. In the gastrocnemius muscle, leucine gavage significantly increased 4E-BP1 phosphorylation (Fig. 7a) but only in the sham and active-Px animals. Following leucine gavage, sham and active-Px animals had an approximately two- and 1.5 -fold increase in $4 \mathrm{E}-\mathrm{BP} 1$ phosphorylation, respectively, compared with water gavage animals $(p<0.05)$. Also, leucine significantly increased S6K1 phosphorylation at threonine 389 (Fig. 7b) but only in the sham and active-Px groups $(p<$ $0.05)$. The sham group had a ninefold increase in S6K1, while the active-Px group had a fourfold increase
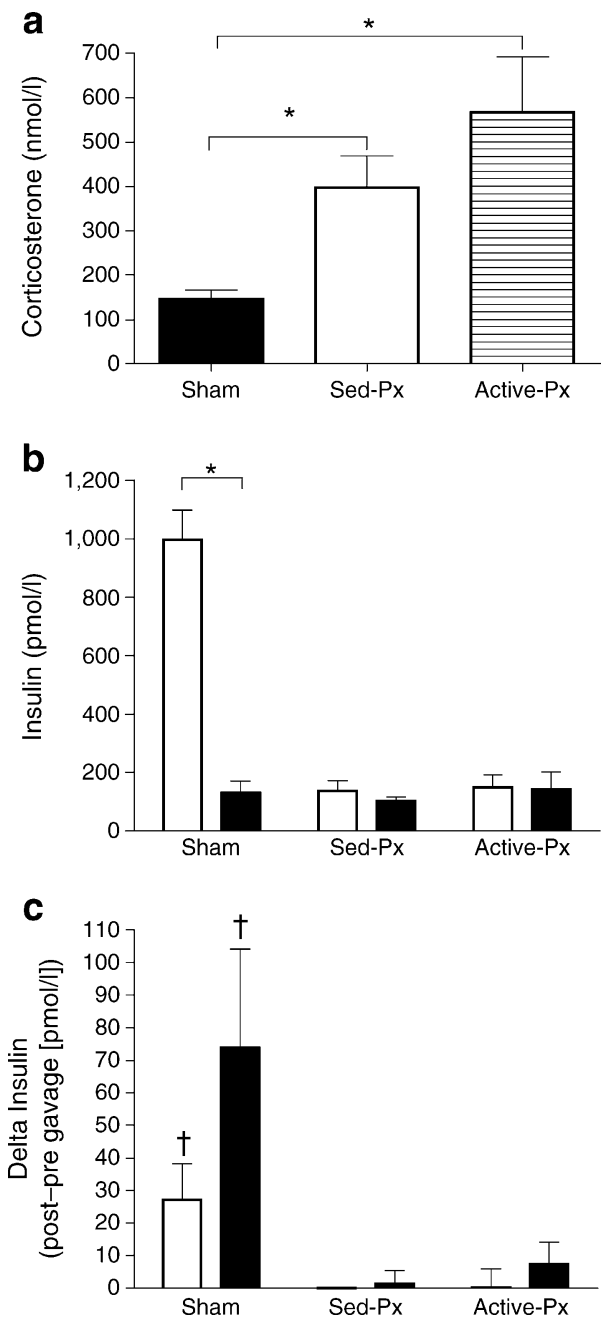

Fig. 3 Corticosterone and insulin levels in sham and Px rats. a Basal plasma corticosterone concentrations were measured from blood samples collected on day 26 (08:00 hours) in the fed state, $n=6-9$ / group. b Fed (white bars) and fasted (black bars) insulin levels were measured from blood samples collected on days 18 and 22 in a separate group of identically treated animals ( $n=5-7 /$ group). c Plasma samples were also collected pre and post gavage on the final experimental day to measure the insulin response to water (white bars) and leucine (black bars) gavage in a subset of animals, $n=5-6 /$ group. ${ }^{*} p<0.05$ for difference between groups. ${ }^{\dagger} p<0.05$ for insulin response to gavage. Values are mean \pm SEM. Sed, sedentary 
a

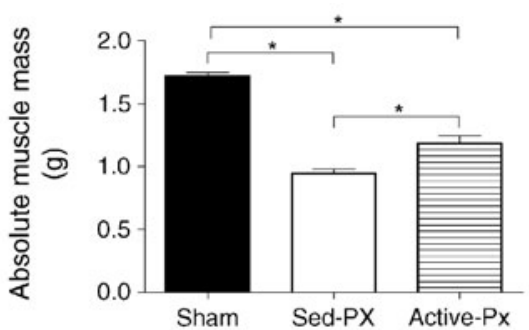

b

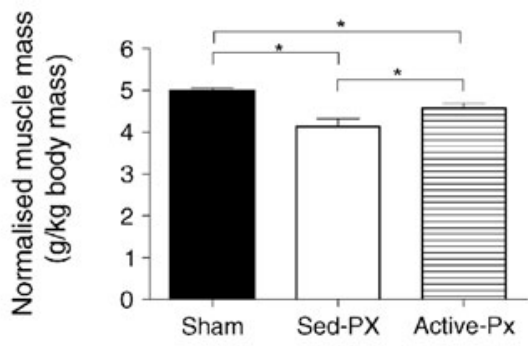

c
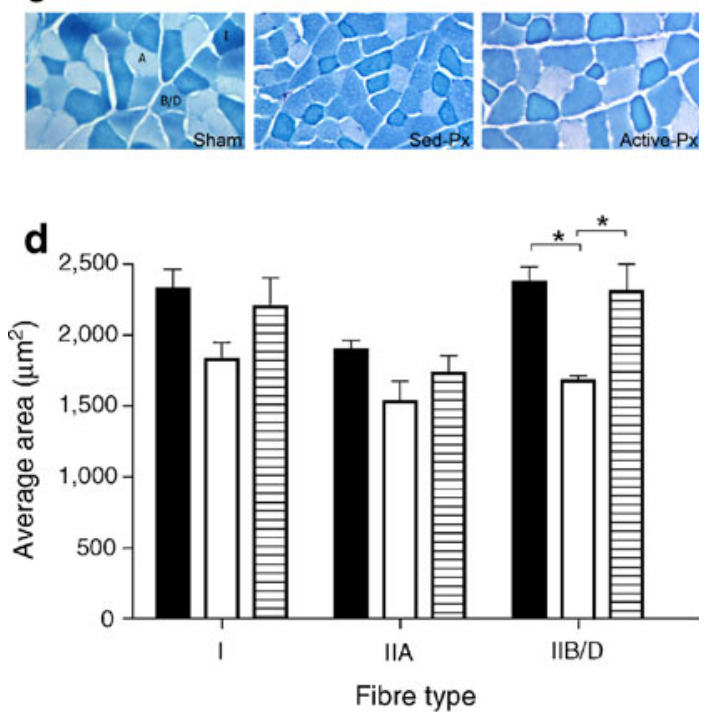

Fig. 4 Absolute and normalised gastrocnemius masses and fibre areas in sham and $\mathrm{Px}$ rats. a Absolute and b normalised gastrocnemius muscle masses were recorded at the time of death to determine differences in muscle mass, $n=11-13 /$ group. A metachromatic myosin ATPase stain was performed on $10 \mu \mathrm{m}$ thick cross sections of gastrocnemius muscle to identify skeletal muscle fibre type. c Representative images of metachromatic fibre type; type I fibres appear dark blue, type IIa appear white and type IIb and IId are not discernible from each other and classified as IIb/d. These fibres appear bluish-purple. d Mean fibre areas, according to fibre type, in gastrocnemius of sham (black bars), sedentary-Px (white bars) and active-Px (hatched bars) ( $n=5-6 /$ group). ${ }^{*} p<0.05$ for difference between groups. Values are mean \pm SEM. A, type IIA fibre; B/D, type IIB/D fibre; I, type I fibre

(significantly different from each other at $p<0.05$ ). In contrast, sedentary-Px animals did not have any significant changes in 4E-BP1 or S6K1 phosphorylation following leucine gavage. To examine if protein degradation/ proteolysis was influenced by hypoinsulinaemia/hyper-

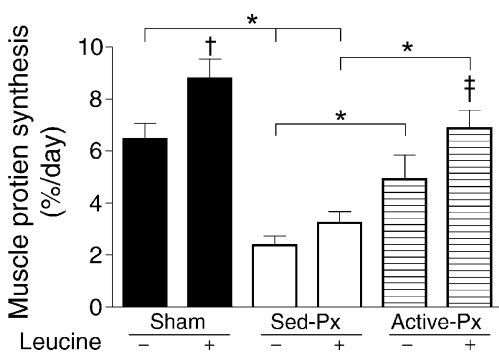

Fig. 5 Muscle fractional protein synthesis rates in gastrocnemius muscle in sham and Px rats. Following an oral gavage of either leucine $(+)$ or water $(-)$, fractional protein synthesis rates were measured, $n=6-9$ /group. ${ }^{*} p<0.05$ for difference between groups. ${ }^{\dagger} p<0.05$ for leucine $(+)$ vs water $(-)$, within each respective animal group. ${ }^{\star} p=0.06$ for leucine $(+)$ vs water $(-)$. Values are mean \pm SEM

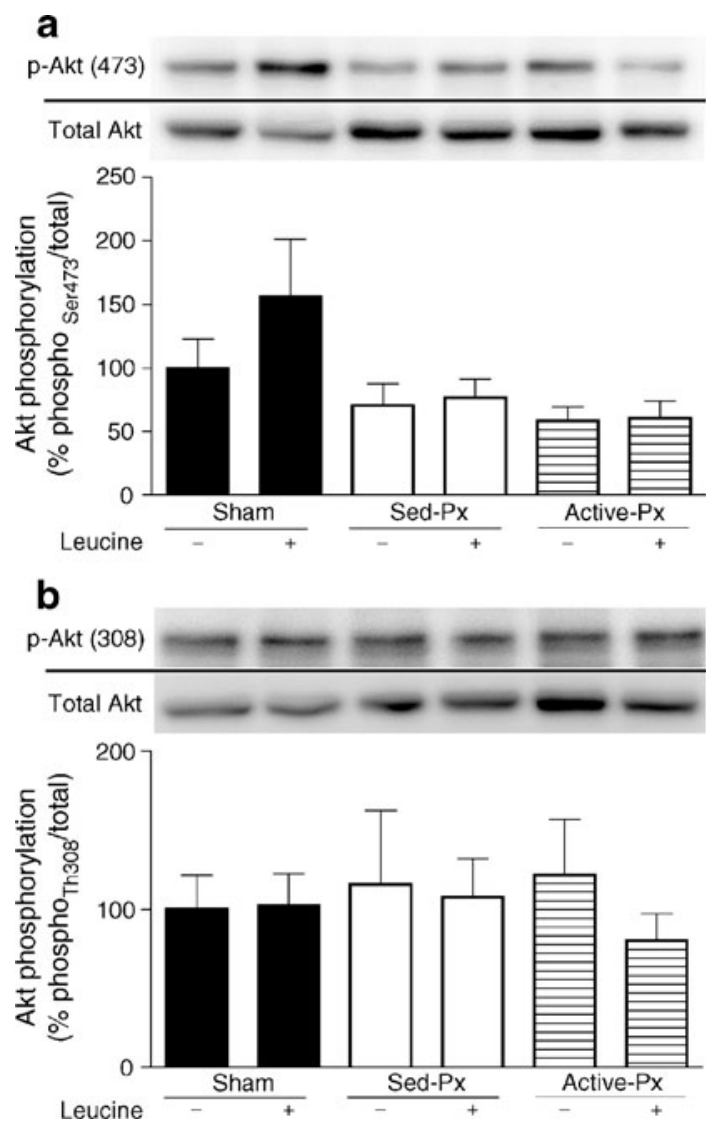

Fig. 6 Akt signalling in sham and Px rats. Phosphorylation of a Akt (serine 473) and b Akt (threonine 308) following a leucine (+) or water $(-)$ gavage in the gastrocnemius muscle of sham, sedentary-Px and activePx groups, $n=6-7 /$ group. All results are relative to $\mathrm{S}(-)$, which represents a normal basal state that has been set to $100 \%$. Values are mean \pm SEM

glycaemia, with and without exercise in our model, we measured total ubiquitinated protein levels in gastrocnemius muscle samples [26]. There were no effects of treatment on this variable (data not shown). 
a

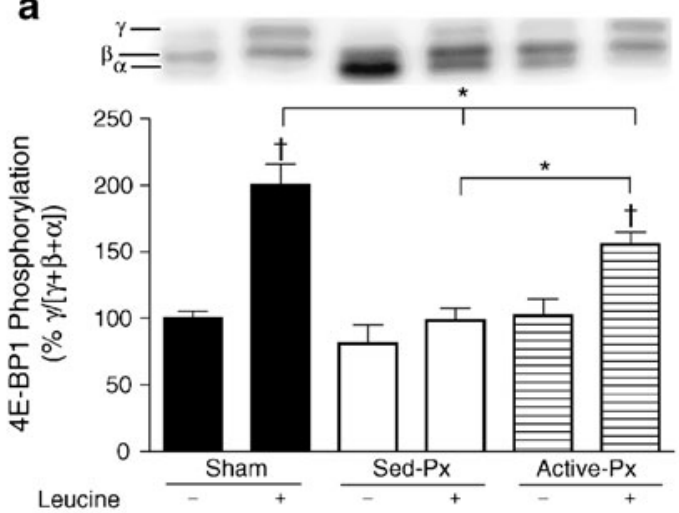

b

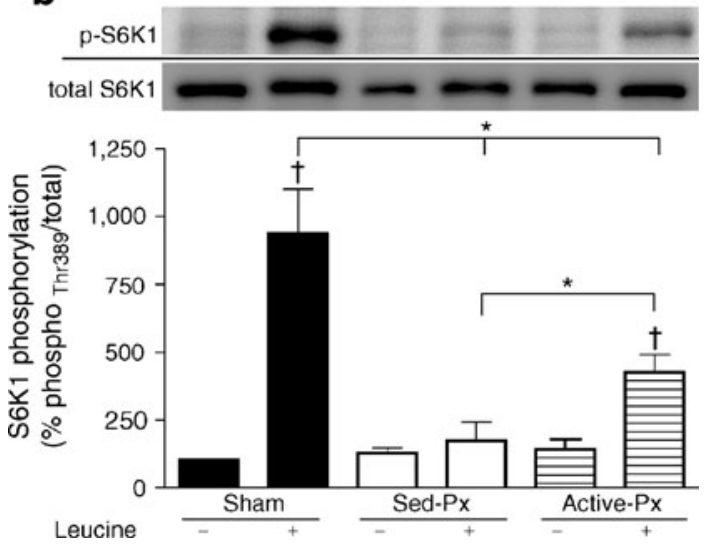

Fig. 7 Markers of mTORC1 signalling in sham and Px rats. Phosphorylation of a 4E-BP1 and b S6K1 (threonine 389) following a leucine (+) or water ( - ) gavage in the gastrocnemius muscle of sham, sedentary-Px and active-Px groups, $n=6-7 /$ group. $\alpha, \beta$, and $\gamma$ indicate the different degrees of phosphorylation of 4E-BP1. All results are relative to S (-), which represents a normal basal state that has been set to $100 \%$. ${ }^{*} p<$ 0.05 for difference between groups. ${ }^{\dagger} p<0.05$ for leucine $(+)$ and water $(-)$ within each respective animal group. Values are mean \pm SEM

\section{Discussion}

The main finding of this study is that, whereas pancreatectomy results in impaired muscle fractional rates of protein synthesis and growth, daily physical activity restores skeletal muscle growth and corrects impaired rates of muscle protein synthesis in young Px rats, without the need for exogenous insulin. Our results also suggest that the mechanism for improved protein synthesis and muscle fibre growth in response to increased physical activity is probably a result of downstream increases in mTORC1 signalling, which are independent of the insulin/IGF1 receptor. Taken together, these results provide sound evidence for the critical role of regular physical activity in helping to normalise impaired skeletal muscle growth and development in a rodent model of adolescent diabetes, when glycaemic control is suboptimal.

Unfortunately for many young people with diabetes, glycaemic control during adolescence deteriorates [5] precisely at the same time when skeletal muscle would normally be rapidly growing [3]. Epidemiological evidence also suggests that youth with diabetes are less physically active and less engaged in sporting activities than their nondiabetic peers [27], which may help to explain, at least in part, their reduced physical capacities [28]. Animal models of diabetes confirm impaired muscle growth $[8,9]$ and reduced rates of muscle protein synthesis when compared with healthy control animals, at least in sedentary cage conditions [9, 11-13, 29]. Surprisingly, a limited number of studies have investigated the effects of regular physical activity on skeletal muscle growth and development in rodent models of adolescent diabetes. Indeed, much of what we know about diabetic myopathy has been from studies published on rodents living in sedentary cage conditions [3, 8-10]. Studies employing resistance type exercise in Px rats $[16,17]$ or via non-physiological ablation of the gastrocnemius to overload the soleus and plantaris muscles in rat models of diabetes [30] show that resistance exercise fails to increase protein synthesis during severe hypoinsulinaemia/hyperglycaemia. In this study, we used a volitional wheel running model to increase levels of aerobic type physical activity in Px rats and examined various markers of muscle protein synthesis in response to nutrient feeding (oral gavage of leucine). Based on our results, we conclude that daily aerobic type exercise is very effective in normalising skeletal muscle growth in diabetic adolescent rats, largely by restoring protein synthesis in response to nutrient feeding. It is important to note that the volume of exercise performed by the animals in this study was considerably less than what had been previously studied using healthy animals of the same age and strain [31]. As such, we believe that even a modest amount of daily physical activity would help normalise muscle growth and metabolism in an animal model of diabetes. However, it is also important to point out that the overall distance ran per day may be slightly more than what is typically observed in treadmill running studies of the same strain $(450-930 \mathrm{~m}$ over a $30 \mathrm{~min}$ training session, 4 days per week) [32]. Unfortunately, we did not measure running speed in this study and thus we are uncertain of the intensity of exercise and thus what muscle fibres were predominantly activated by the activity, nor can we determine whether the major stimulus for protein synthesis was running distance or running speed. How this volume and type of exercise might translate to adolescents with diabetes is also unclear.

One surprising finding in this study is that, despite improvements in glycaemia and in muscle protein synthesis, we did not observe improvements in body mass gains in the active-Px rats (Fig. 1). Since the reduced body mass caused by diabetes was not restored by increased exercise in our model, even though there was restoration in 
muscle growth, we speculate that the increase in energy expenditure caused by the increased activity might have been provided by the oxidation of lipids, thus promoting an altered body composition rather than weight gain per se (i.e. high lean mass, low fat mass).

Consistent with previous results $[8,9,33,34]$, our muscle fibre analysis showed that sedentary behaviour in diabetic rats results in a fibre type-specific reduction in cross-sectional area, with the type IIb/d fibres being most compromised. In addition, although effective in attenuating muscle fibre area loss in all fibre types, we show that regular voluntary physical activity in diabetic animal models largely protects these same glycolytic muscle fibres. Since sedentary cage living conditions generally recruit only type I and IIa muscle fibres, it may be that the effectiveness of increased physical activity on muscle protein synthesis results from the recruitment of the glycolytic fibres, along with a greater activation of the more oxidative fibres. Whether aerobic type exercise is more effective than resistance type exercise in accruing skeletal muscle mass in the pancreatectomy model remains to be determined.

Oral administration of leucine is commonly used in animal models to simulate feeding-induced changes in protein synthesis [35-37] and to show that sedentary diabetic organisms have impaired protein synthesis rates when compared with control animals [9]. Here we show that a modest amount of aerobic physical activity increases both the basal (fasting) and nutrient-stimulated rates of muscle protein synthesis in severely hyperglycaemic Px animals without an insulin response to feeding. In fact, our findings illustrate that, in response to leucine gavage, diabetic rats that are physically active but non-insulintreated have protein synthesis levels that are comparable with healthy sedentary sham rats. It is important to note, however, that Px rodents do have some low level of circulating insulin, as would be expected since $10 \%$ of the pancreas was left intact (Fig. 3b). This level of basal insulin is similar to other rodent models of type 1 diabetes such as the Akita mouse $[8,38]$ or the streptozotocininduced diabetic rodent $[8,39,40]$. Considering that these diabetic animals ran considerably less than what is typically observed in healthy animals of the same age/strain ( $2.2 \mathrm{vs} 9 \mathrm{~km}$ per night) [31], these findings provide strong evidence for the anabolic effect of aerobic type exercise on leucine stimulated protein synthesis in the skeletal muscle of diabetic rat models. While a previous report by Anthony et al. showed that an acute leucine feeding of $1.35 \mathrm{~g} / \mathrm{kg}$ increases muscle protein synthesis in sedentary diabetic rodents [41], we show increased protein synthesis in active diabetic animals using a much lower amount of leucine $(0.48 \mathrm{~g} / \mathrm{kg})$. This is important when considering the future feasibility of using leucine as a nutritional strategy to normalise muscle growth in humans with the disease [20].

Although acute leucine administration may increase insulin levels if given in moderate to high doses $(\geq 0.675 \mathrm{~g} \mathrm{~L}$-leucine $/ \mathrm{kg}$ body weight $)$ in healthy rodents [20], leucine gavage in this study did not appear to increase insulin levels beyond water gavage (Fig. 3c), nor Akt phosphorylation (Fig. 6a,b), at least not in the Px groups. Thus, it would appear that the protein synthesis effects of regular exercise, coupled with low dose leucine feeding, cannot be explained by changes in plasma insulin levels nor insulin signalling. These observations are consistent with the findings of Spangenburg et al. [19] who reported that functional muscle overload does not require insulin/IGF-1 signalling to promote muscle hypertrophy and S6K1 activation. Furthermore, we show here that the exercise-induced improvement in muscle protein synthesis occurred at steps distal to Akt activation. This observation is consistent with mTORC1 activation and stimulation of cap-dependent mRNA translation [42]. Taken together, these data suggest that insulin signalling through Akt may not be required to increase protein synthesis in diabetic rat models, as long as exercise is performed. It is important to note, however, that basal protein synthesis in active-Px rats (i.e. water gavage) was higher than in sedentary-Px rats, without observable increases in Akt, 4E-BP1 or S6K1 phosphorylation. It is possible, therefore, that exercise itself might stimulate protein synthesis completely independently of mTORC1 signalling. Previous work by Williamson et al. [43] has also shown that, following acute endurance exercise, mTORC1 signalling to biomarkers of translation initiation was repressed. Furthermore, they showed no measurable effects of endurance exercise on Akt phosphorylation, but showed increases in both extracellular signal-regulated kinase (ERK) $1 / 2$ and eIF4E phosphorylation. Taken together, these results suggest that physical activity may regulate muscle protein synthesis through signalling pathways that are independent of the mTORC1 network [44], although this hypothesis would require additional experimentation with selective inhibitors of the insulin signalling cascade (e.g. wortmannin) and/or mTORC1/S6K1 activation (e.g. rapamycin).

\section{Conclusions}

In summary, we found that voluntary wheel running is effective in stimulating protein synthesis in severely diabetic Px animal models devoid of exogenous insulin therapy. In addition, with regular activity, diabetic animal models show an improved mTORC1 signalling response and a higher rate of fractional protein synthesis in muscle in response to leucine feeding. These findings may have 
important clinical implications for the role of regular exercise in helping to normalise muscle growth and development during periods of hypoinsulinaemia in adolescents with type 1 diabetes.

Acknowledgements This research was supported by grants from the Natural Sciences and Engineering Research Council (NSERC) of Canada (to M.C. Riddell and O.A. Adegoke) and the Canadian Diabetes Association (M.C. Riddell). We thank S. Bonner-Weir for instructing us on how to perform the $90 \%$ pancreatectomy surgery in rats and $\mathrm{M}$. Connor for allowing us to use his Kodak imaging station for our protein abundance analysis.

Contribution statement All of the authors listed helped with the conception/design of the study as well as the analysis and/or interpretation of these data. All authors assisted in either drafting the article and/or revising it critically. Finally, all authors approved the final version of the manuscript for publication.

Duality of interest The authors declare that there is no duality of interest associated with this manuscript.

\section{References}

1. Caso G, McNurlan MA (2010) Effect of insulin on whole body protein metabolism in children with type 1 diabetes. Curr Opin Clin Nutr Metab Care 13:93-96

2. Charlton M, Nair KS (1998) Protein metabolism in insulindependent diabetes mellitus. J Nutr 128:323S-327S

3. Krause MP, Riddell MC, Hawke TJ (2011) Effects of type 1 diabetes mellitus on skeletal muscle: clinical observations and physiological mechanisms. Pediatr Diabetes 12:345-364

4. Diabetes Control and Complications Trial Research Group (1994) Effect of intensive diabetes treatment on the development and progression of long-term complications in adolescents with insulindependent diabetes mellitus: Diabetes Control and Complications Trial. J Pediatr 125:177-188

5. Hamilton J, Daneman D (2002) Deteriorating diabetes control during adolescence: physiological or psychosocial? J Pediatr Endocrinol Metab 15:115-126

6. Ceriello A, Ihnat MA, Thorpe JE (2009) Clinical review 2: the "metabolic memory": is more than just tight glucose control necessary to prevent diabetic complications? J Clin Endocrinol Metab 94:410-415

7. Braziuniene I, Garlick J, Mileva I, Desikan V, Wilson TA, McNurlan M (2009) Effect of insulin with oral nutrients on whole-body protein metabolism in growing pubertal children with type 1 diabetes. Pediatr Res 65:109-112

8. Krause MP, Riddell MC, Gordon CS, Imam SA, Cafarelli E, Hawke TJ (2009) Diabetic myopathy differs between Ins2Akitat/- and streptozotocin-induced type 1 diabetic models. J Appl Physiol 106:1650-1659

9. Gordon CS, Serino AS, Krause MP et al (2010) Impaired growth and force production in skeletal muscles of young partially pancreatectomized rats: a model of adolescent type 1 diabetic myopathy? PLoS One 5:e14032

10. Krause MP, Moradi J, Nissar AA, Riddell MC, Hawke TJ (2011) Inhibition of plasminogen activator inhibitor-1 restores skeletal muscle regeneration in untreated type 1 diabetic mice. Diabetes 60:1964-1972

11. Pain VM, Albertse EC, Garlick PJ (1983) Protein metabolism in skeletal muscle, diaphragm, and heart of diabetic rats. Am J Physiol 245:E604-E610
12. Pain VM, Garlick PJ (1974) Effect of streptozotocin diabetes and insulin treatment on the rate of protein synthesis in tissues of the rat in vivo. J Biol Chem 249:4510-4514

13. Ashford AJ, Pain VM (1986) Effect of diabetes on the rates of synthesis and degradation of ribosomes in rat muscle and liver in vivo. J Biol Chem 261:4059-4065

14. Johnston AP, Campbell JE, Found JG, Riddell MC, Hawke TJ (2007) Streptozotocin induces G2 arrest in skeletal muscle myoblasts and impairs muscle growth in vivo. Am J Physiol Cell Physiol 292:C1033-C1040

15. Little JP, Phillips SM (2009) Resistance exercise and nutrition to counteract muscle wasting. Appl Physiol Nutr Metab 34:817828

16. Fedele MJ, Hernandez JM, Lang CH et al (2000) Severe diabetes prohibits elevations in muscle protein synthesis after acute resistance exercise in rats. J Appl Physiol 88:102-108

17. Kostyak JC, Kimball SR, Jefferson LS, Farrell PA (2001) Severe diabetes inhibits resistance exercise-induced increase in eukaryotic initiation factor 2B activity. J Appl Physiol 91:79-84

18. Bolster DR, Jefferson LS, Kimball SR (2004) Regulation of protein synthesis associated with skeletal muscle hypertrophy by insulin-, amino acid- and exercise-induced signalling. Proc Nutr Soc 63:351-356

19. Spangenburg EE, Le Roith D, Ward CW, Bodine SC (2008) A functional insulin-like growth factor receptor is not necessary for load-induced skeletal muscle hypertrophy. J Physiol 586:283-291

20. Crozier SJ, Kimball SR, Emmert SW, Anthony JC, Jefferson LS (2005) Oral leucine administration stimulates protein synthesis in rat skeletal muscle. J Nutr 135:376-382

21. Brown AP, Dinger N, Levine BS (2000) Stress produced by gavage administration in the rat. Contemp Top Lab Anim Sci 39:17-21

22. Garlick PJ, McNurlan MA, Preedy VR (1980) A rapid and convenient technique for measuring the rate of protein synthesis in tissues by injection of $[3 \mathrm{H}]$ phenylalanine. Biochem J 192:719723

23. Zargar S, Moreira TS, Samimi Seisan H et al (2011) Skeletal muscle protein synthesis and the abundance of the mRNA translation initiation repressor PDCD4 are inversely regulated by fasting and refeeding in rats. Am J Physiol Endocrinol Metab 300 (6):E986-E992

24. Jepson MM, Pell JM, Bates PC, Millward DJ (1986) The effects of endotoxaemia on protein metabolism in skeletal muscle and liver of fed and fasted rats. Biochem J 235:329-336

25. Ogilvie RW, Feeback DL (1990) A metachromatic dye-ATPase method for the simultaneous identification of skeletal muscle fiber types I, IIA, IIB and IIC. Stain Technol 65:231-241

26. Wing SS, Haas AL, Goldberg AL (1995) Increase in ubiquitinprotein conjugates concomitant with the increase in proteolysis in rat skeletal muscle during starvation and atrophy denervation. Biochem J 307(Pt 3):639-645

27. Valerio G, Spagnuolo MI, Lombardi F, Spadaro R, Siano M, Franzese A (2007) Physical activity and sports participation in children and adolescents with type 1 diabetes mellitus. Nutr Metab Cardiovase Dis 17:376-382

28. Riddell MC, Iscoe KE (2006) Physical activity, sport, and pediatric diabetes. Pediatr Diabetes 7:60-70

29. Flaim KE, Copenhaver ME, Jefferson LS (1980) Effects of diabetes on protein synthesis in fast- and slow-twitch rat skeletal muscle. Am J Physiol 239:E88-E95

30. Goldberg AL (1968) Role of insulin in work-induced growth of skeletal muscle. Endocrinology 83:1071-1073

31. Fediuc S, Campbell JE, Riddell MC (2006) Effect of voluntary wheel running on circadian corticosterone release and on HPA axis responsiveness to restraint stress in Sprague-Dawley rats. J Appl Physiol 100:1867-1875 
32. Winder WW, Yang HT, Arogyasami J (1988) Liver fructose 2,6bisphosphate in rats running at different treadmill speeds. Am $\mathbf{J}$ Physiol 255:R38-R41

33. Medina-Sanchez M, Rodriguez-Sanchez C, Vega-Alvarez JA, Menedez-Pelaez A, Perez-Casas A (1991) Proximal skeletal muscle alterations in streptozotocin-diabetic rats: a histochemical and morphometric analysis. Am J Anat 191:48-56

34. Klueber KM, Feczko JD (1994) Ultrastructural, histochemical, and morphometric analysis of skeletal muscle in a murine model of type I diabetes. Anat Rec 239:18-34

35. Norton LE, Layman DK, Bunpo P, Anthony TG, Brana DV, Garlick PJ (2009) The leucine content of a complete meal directs peak activation but not duration of skeletal muscle protein synthesis and mammalian target of rapamycin signaling in rats. J Nutr 139:1103-1109

36. Kimball SR, Jefferson LS (2006) Signaling pathways and molecular mechanisms through which branched-chain amino acids mediate translational control of protein synthesis. J Nutr 136:227S-231S

37. Kimball SR, Jefferson LS (2004) Regulation of global and specific mRNA translation by oral administration of branchedchain amino acids. Biochem Biophys Res Commun 313:423-427

38. Mathews CE, Langley SH, Leiter EH (2002) New mouse model to study islet transplantation in insulin-dependent diabetes mellitus. Transplantation 73:1333-1336
39. Akirav EM, Chan O, Inouye K, Riddell MC, Matthews SG, Vranic M (2004) Partial leptin restoration increases hypothalamicpituitary-adrenal activity while diminishing weight loss and hyperphagia in streptozotocin diabetic rats. Metabolism 53:1558-1564

40. Chan O, Chan S, Inouye K, Shum K, Matthews SG, Vranic M (2002) Diabetes impairs hypothalamo-pituitary-adrenal (HPA) responses to hypoglycemia, and insulin treatment normalizes HPA but not epinephrine responses. Diabetes 51:1681-1689

41. Anthony JC, Reiter AK, Anthony TG et al (2002) Orally administered leucine enhances protein synthesis in skeletal muscle of diabetic rats in the absence of increases in 4E-BP1 or S6K1 phosphorylation. Diabetes 51:928-936

42. Hay N, Sonenberg N (2004) Upstream and downstream of mTOR. Genes Dev 18:1926-1945

43. Williamson DL, Kubica N, Kimball SR, Jefferson LS (2006) Exercise-induced alterations in extracellular signal-regulated kinase $1 / 2$ and mammalian target of rapamycin (mTOR) signalling to regulatory mechanisms of mRNA translation in mouse muscle. J Physiol 573:497-510

44. Drummond MJ, Dreyer HC, Fry CS, Glynn EL, Rasmussen BB (2009) Nutritional and contractile regulation of human skeletal muscle protein synthesis and mTORC1 signaling. J Appl Physiol $106: 1374-1384$ 